\title{
Towards a Narrative-Aware Design Framework for Smart Urban Environments
}

\author{
Lara Srivastava ${ }^{1, *}$ and Athena Vakali ${ }^{2, * *}$ \\ ${ }^{1}$ Department of Media Communications, Webster University, Geneva, Switzerland \\ lara.srivastava@gmail.com \\ ${ }^{2}$ Department of Informatics, Aristotle University of Thessaloniki, Greece \\ avakali@csd.auth.gr
}

\begin{abstract}
Innovation in smart city systems is based on the principle that devices, places and everyday things can each be enabled to serve people in a real-time and responsive manner. This chapter presents a novel approach to the design of smart city systems that takes into account not only technical installations in a future Internet of Things environment, but also the power of human storytelling in an always-on networked world. It is only when environments are both sensor-driven and socially-aware that a more holistic, and therefore more useful, urban narrative can emerge in the future Internet context. The present chapter proposes a new narrative-aware design framework and applies it to a hypothetical city scenario in order to highlight its main components and the benefits it may offer to a future Internet city's actors.
\end{abstract}

Keywords: Smart cities, sensor data analysis, social data mining, smart urban services, Internet of things, narrative, storytelling, navigation, mobility, sensors, web 2.0.

\section{Introduction}

The Internet of today enables users to access an unprecedented amount of information at anytime and from any device. In the future, an emerging Internet of Things (IoT) will connect everyday objects (such as toothbrushes, shoes or car keys), which will become information storehouses of their own, capable of collecting and transmitting real-time data to their surrounding environment (people, places and things). The resulting myriad of smart interconnected objects and places will make up the intelligent urban landscape of the future.

Urban environments offer unique opportunities for developing and testing new applications and platforms in line with the vision of the Internet of Things. European IoT platforms have already begun emerging over the last few years inline with the

\footnotetext{
Professor.

** Associate Professor. 
future Internet momentum. Large smart city infrastructures have now been set up in Europe (e.g.SmartSantander ${ }^{1}$, Spain) and worldwide (e.g. Songdo ${ }^{2,}$ South Korea).

The growing need and interest in smart city innovation was highlighted by the Commission in its report "Internet of Things in 2020: A roadmap for the future", which it identified key topics such as "Smart living" as part of what it termed a "mastered continuum of people, computers and things". There is a growing number of innovative social and people-centric application areas, including social networking, smart metering, smart data collection, city information models and so on [Atzoria10].

Although these application areas provide an excellent starting point to test services and infrastructure, most offer merely quantitative solutions for a world that is primarily qualitative (particularly from the human perspective). For the most part, they collect and store data and information from technical devices and sensors. With the growth of web 2.0 and social media, however, a wide array of human experience, information and know-how is being shared and distributed across networks information that has yet to be properly harvested for the creation of smarter living environments.

The present chapter proposes a new design framework for the smart city, one which considers quantitative sensor-generated data as well as qualitative human-generated data through participatory web platforms, in the future Internet context. In this manner, storytelling and "listening" by networked objects is enhanced and vetted by human storytelling, thereby getting us that much closer to true human-machine collaboration.

This chapter begins with an overview and gap analysis of the main developments in urban IoT applications with a focus on resident mobility (Section 2). It then goes on to highlight the need for a new kind of holistic urban storytelling (Section 3). The section that follows describes a new design approach for smart urban environments that is both sensor-driven and socially aware (Section 4). The concept is then applied to a hypothetical urban mobility scenario (Section 5).

\section{Urban Mobility: State of the Art and Gap Analysis}

Smart city platforms and installations continue to expand as IoT innovations emerge and services develop. At the same time, urban residents continue to multiply the number of mobile devices they use, and through social media, have become important generators of content themselves (with varying levels of objectivity). As mentioned above, applications can benefit from the combination of smart city sensors and data from users on the move.

Mobility services can capture the pulse and momentum of a city, through sensors, status updates, and tracking. Thus far, urban mobility solutions have relied primarily on information and communication technology to manage transport networks and

\footnotetext{
${ }^{1}$ http: / / www. smartsantander.eu/

2 http: / /www. songdo.com/

3 ftp: / / ftp.cordis. europa.eu/pub/fp7/ict/docs/enet/internet-ofthings-in-2020-ec-eposs-workshop-report-2008-v3_en.pdf
} 
Table 1. Urban mobility: Current and future requirements

\begin{tabular}{|c|c|c|}
\hline Area & Current developments & Future Needs and Requirements \\
\hline $\begin{array}{l}\text { sensor-aware } \\
\text { transport } \\
\text { management }\end{array}$ & $\begin{array}{l}\text { - Lower hazardous emis- } \\
\text { sions from city traffic } \\
\text { - Environmental, accurate } \\
\text { and cost-efficient road } \\
\text { traffic management }\end{array}$ & $\begin{array}{l}\checkmark \text { monitor city traffic based on } \\
\text { multiple inputs (sensors and } \\
\text { social streams) } \\
\checkmark \text { leverage real time feedback } \\
\text { from residents on the move) }\end{array}$ \\
\hline $\begin{array}{l}\text { urban } \\
\text { travel } \\
\text { planners }\end{array}$ & $\begin{array}{l}\text { - plan navigations from A to } \\
\text { B by using particular } \\
\text { location and transport } \\
\text { modes. } \\
\text { - Generic planning } \\
\text { recommendations on pre- } \\
\text { defined fixed city's } \\
\text { touristic spots. }\end{array}$ & $\begin{array}{l}\checkmark \text { increase personalized urban } \\
\text { travel planning aware of both } \\
\text { environmental and residents } \\
\text { status } \\
\checkmark \text { provide safe residents' mobility } \\
\text { by data integration }\end{array}$ \\
\hline $\begin{array}{l}\text { social-wise } \\
\text { urban mobility } \\
\text { guidance }\end{array}$ & $\begin{array}{l}\text { - } \text { social networking } \\
\text { supports urban mobility } \\
\text { primarily by POI and } \\
\text { user's ratings } \\
\text { - location based social } \\
\text { networks offline analysis }\end{array}$ & $\begin{array}{l}\checkmark \text { leverage Web } 2.0 \text { capabilities to } \\
\text { allow residents expressing } \\
\text { their city sensing } \\
\checkmark \text { exploit active and real-time } \\
\text { sensor /social capabilities } \\
\text { for urban notifications and } \\
\text { alerting }\end{array}$ \\
\hline
\end{tabular}

guide users through a city. Examples include European projects such as SMILE ${ }^{4}$ which deals with sustainable and cost-efficient mobility and i-Travel ${ }^{5}$ which provides personalized, context-aware "virtual travel assistant" services in urban settings.

The majority of existing ICT urban mobility applications have focused on :

- Sensor-aware transport: This area deals with effective traffic management for a city's public transportation system. Sensors (e.g. in combination with IoT platforms) capture specific measurements (such as $\mathrm{CO} 2$ emissions) [6]. Use cases have focused on managing city traffic, eco-driving and emergency handling [9].

- Urban travel planners: Planners are generated on the basis of the current location of users, their preferences and mobile device settings. Semantic web tools and technologies such as Global Navigation Satellite Systems (GNSS) and Geographical Information Systems (GIS) are used to improve context and geolocation awareness, respectively [12]. Current mobile route planning tools are typically geared towards points of interest for tourists (sightseeing, hotels, restaurants, and packaged tour routes) [14], [17].

${ }^{4}$ SMILE : Towards Sustainable Mobility for people in urban areas http : / /www. istworld.org/ProjectDetails.aspx? Project Id $=258180 \mathrm{ce} 08 \mathrm{fd} 44 \mathrm{cfa} 050 \mathrm{fC}$ $554 c 80 e 828$

5 i-Travel : The connected traveler in the city, region and world of tomorrow http:// cordis. europa.eu / fetch?CALLER=FP7_PROJ_EN\&ACTION=D\&RCN=85751 
- Social-wise urban mobility guidance: With the emergence of web 2.0, some urban mobility applications have sought to leverage the opinions of users on urban points of interest (POIs). Collaborative filtering approaches are combined with locationbased partitioning and user comments [15]. More recently, recommendations for POIs have used location-based social networks (e.g. Foursquare) and included user ratings, proximity and similarities [16], [13]. Services such as GeoLife analyze the GPS trajectories of users off-line to provide personalized recommendations [18].

Table 1 summarizes current practices in the three areas identified above. It identifies some of the more pressing needs and priorities for each area. It would seem evident that little effort has been made to date to exploit the synergies between technical data and social data streams.

It is important to note that both the IoT industry and the mobile industry are continuing to expand, though not always in the same direction. Therefore, much is to be gained by unifying their vision and creating a more holistic understanding of user needs and requirements. The convergence of machine and human perspectives will serve to enrich and facilitate daily living in the urban context. The next sections propose a new design for urban mobility based on this principle.

The needs and requirements (summarized in Table 1) reveal that a proposed approach which will merge real-time social and sensor data streams is expected to be beneficial since citizens engagement can be improved. Such an improvement is guaranteed by the fact that, according to the authors knowledge, there are no universal applications which go beyond a typical residents navigation or mobility assistance.

\section{Towards a Richer Form of Urban Narrative}

In an Internet of Things environment, individual objects collect data through a combination of sensor and location tagging. The way these objects then transmit information about the world around them helps people begin to create an urban narrative that spans both space and time. In other words, these bits and bytes contribute to the "digital memory" of a place, a city, or an event. Such digital memories are facilitated in the future Internet context.

However, the information revealed by sensors provides only half the picture. It is limited to data that devices have gathered through technical means- the sounds they hear, the temperature they detect. But if sensor data is then combined with the many layers of human observation and perception (human storytelling), what results is a richer, more holistic "urban digital narrative". For not only will sensors report back with real-time recorded data, but these reports will be further checked and enhanced by the human perspective. This will have the effect of expanding the role of sensors beyond mere data-listeners. By way of analogy, sensors will no longer only hear the "words", but they will also be able to "listen" to the stories; they will not only collect fragments of data, but be part and parcel of a larger human picture. 


\subsection{Sensor Data}

Today's urban Internet of Things infrastructure consists mainly of automatic identification and sensor installations connected with local, global, public and private networks.

These networked sensors are widely used in the context of smart cities in order to accurately measure and record physical parameters and phenomena that have an incidence on the life of residents. They automatically listen to the environment in which they are placed according to how they are programmed. With the right analytical methods, this data can be used to identify and predict when special conditions or phenomena arise, e.g. traffic congestion or atmospheric pollution. In turn, this information can assist authorities in taking short-term (e.g inform/alert residents and especially vulnerable social groups) or long-term action (e.g. understanding the progress of the phenomena and trying to address them in order to improve the city's conditions).

Even smart sensor networks that capture information about the technical parameters of a given phenomenon cannot share or combine this information with the perception or mood of residents, and are thus far from creating a complete urban digital story. In the future Internet reality, there is a need for completing existing smart systems with people-engaged narratives that are not only technical but social in nature, where the objective is not only to listen to the "data", but to support the exchange (story-listening and story-telling) of urban "stories".

\subsection{Social Stories}

The daily social activities of residents are being broadcast in real-time by a growing number of mobile devices. Urban residents use mobile devices to manage their professional and personal lives, their interaction with others, and their interaction with their environment. Not surprisingly, the use of web 2.0 applications and social media has proliferated on mobile networks. In this context, mobile users act as storytellers and listeners, exchanging experiences over the internet. This so-called "urban social pulse" can be gleaned through applications like Flickr, Facebook, Twitter but also location-based services such as Foursquare and Gowalla. It contributes to a larger urban story that can be heard by authorities and residents. For instance, residents might express overcrowding and excessive heat (e.g. at a concert), and this might serve to override physical data such as room temperature (which may not be high enough to cause concern). Future concerts planned in that area could be reconsidered. In this manner, a future internet would augment sensor-generated data into usable stories that might refine a resident-driven urban narrative.

\subsection{From Data Listening to Story Telling}

Figure 1 is a graphical representation of the two sides of an urban story (sensor data and resident data) and how these fit within the smart city context. 


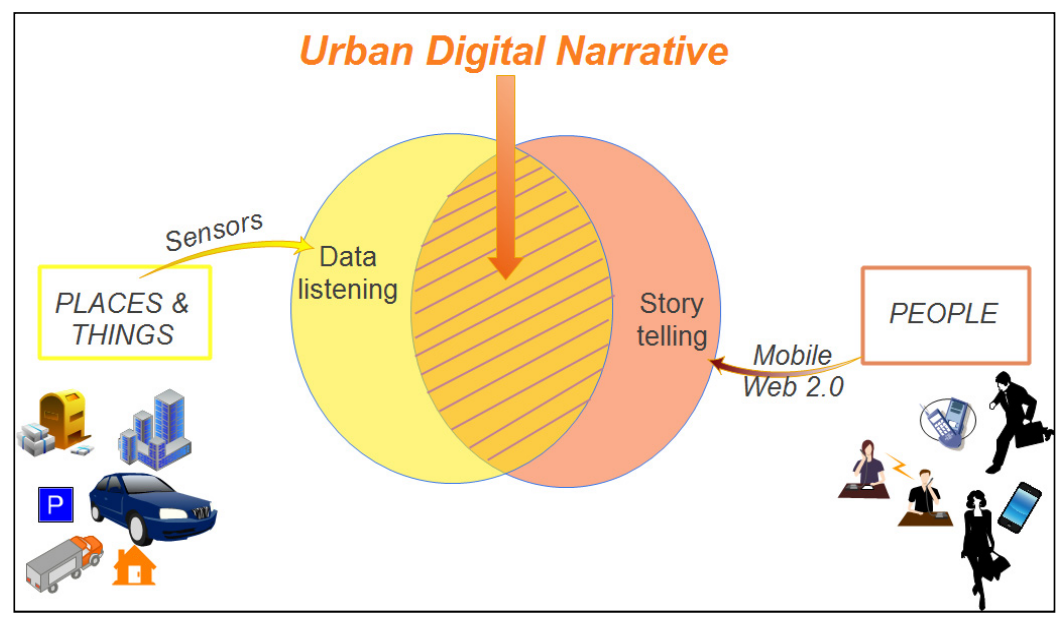

Fig. 1. Urban data listening and story telling

As illustrated, the storytelling of residents on the move can occur in real-time through the use of mobile devices and web 2.0 platforms. Simultaneously, and also in real-time, sensors listen to things and detect environmental conditions (in various places). A clearer and richer urban digital narrative emerges only at the intersection of these two data streams. Smart urban applications that can listen to this more complete narrative are likely to provide richer, more people-centric and context-aware services.

Without a doubt, the time is ripe to take urban mobility services this important step further. The notion of context-awareness in the future internet need not be limited to sensory data or machine-generated information, but must also leverage the power of human storytelling.

\subsection{Uses and Benefits}

In the future internet, sensor and socially-aware storytelling could provide vital support and guidance to a city's actors, such as residents, visitors and authorities. Uses and benefits for these actors are highlighted below:

- Residents typically move around their city on a daily basis using different modes of transport (e.g. personal vehicles, public transport, walking, running, biking) and for various purposes at different times of the day (e.g. work, caregiving, errands, leisure etc.). Narrative-aware services should place emphasis on the collection of information that generates real-time adaptive recommendations for residents. This can improve navigation within the city and can assist in the selection of the most appropriate routes based on various parameters, e.g. distance, $\mathrm{CO} 2$ emissions, congestion, noise levels, parking, public transport routes and schedules. Resident input (stories) over social media can provide invaluable qualitative information to complement sensor-generated data. 
- Visitors are particularly interested in city navigation, POIs, queues and crowds. Narrative-aware services could offer recommendations on points of interest or city walks, based on proximity, popularity, tourist opinions, weather, opening times, congestion and so on. Both residents and visitors could provide observations that would be used to complement any technical measurements taken by sensors and location-based technologies.

- Authorities (such as the police, fire department, or city council) could exploit narrative-aware urban services and applications to enable monitoring of the city's major "variables" (e.g. noise, temperature, crime, $\mathrm{CO} 2$ levels) through global and user-centered visualization interfaces. Such interfaces could also enable detection of vulnerable geographical areas which indicate both over-threshold sensor measurements and any alerts broadcast by the residents on the move.

The fact that the proposed narrative approach is flexible and multi-scenario oriented, differentiates it from existing approaches which are more vertical and they focus on improving the separate angles, i.e. either the data management or the usage side. The end-users involvement is expected from the appealing story telling emphasis which is expected to attract in particular users with mobile phones in a smart city context.

\section{The Urban Narrative-Aware Design Framework}

Capturing and reading urban narratives involves several complex steps and processes, and cuts across various service layers (infrastructure applications, content, usage). The design of such a framework must be cognizant of this complexity. Figure 2 is an illustration of the urban narrative-aware design framework, with functionality at three different levels:

- Data and stories: All tasks related to the collection of data and socially-generated stories are carried out at this level. Differentiated techniques are required for storing sensor data and social streams into individual "DataStores" or data repositories. Targeted data storage and scalable indexing schemes should be used to cope with the ever-growing number of sensor and social measurements. Moreover, specific data and stories pre-processing is required to provide noise-free DataStores.

- Analysis and Processing. DataStore integration, refinement and analysis are key tasks at this level. In particular, the first core task is the integration of sensor data and human stories, with the objective of constructing new narrative-aware DataStores, i.e. "Narrative stores" or "NarraStores". NarraStores host the various digital narratives of an urban context. This integration is an ongoing task which can benefit from regular refinement (i.e. calibration) and analysis, due to the emerging and unpredictable nature of urban sensor and social data streams. DataStore calibration involves processes which will validate and fine-tune information from the two different data sources (sensors and social) and will revise either the content of the DataStore or the data collection process itself. DataStore analysis can involve a wide array of methodologies and algorithms from the fields of data 
mining and recommender systems. This leads to the generation of quantitative and qualitative NarraStores. The continuous calibration and analysis processes optimize the content of NarraStores by summarizing, clustering and packaging diverse narratives.

- Services and applications. NarraStores are the foundation upon which a wide variety of services and applications can be built. The proposed design in Fig.2 puts forward an initial number of key urban services (assistance, alerting, and planning) for the main urban actors (as outlined in 3.4). Such services leverage the Narrative stores at their disposal in order to offer contextualized mobile and Web applications for short-term (e.g. alerting) or long-term tasks (e.g. business planning).

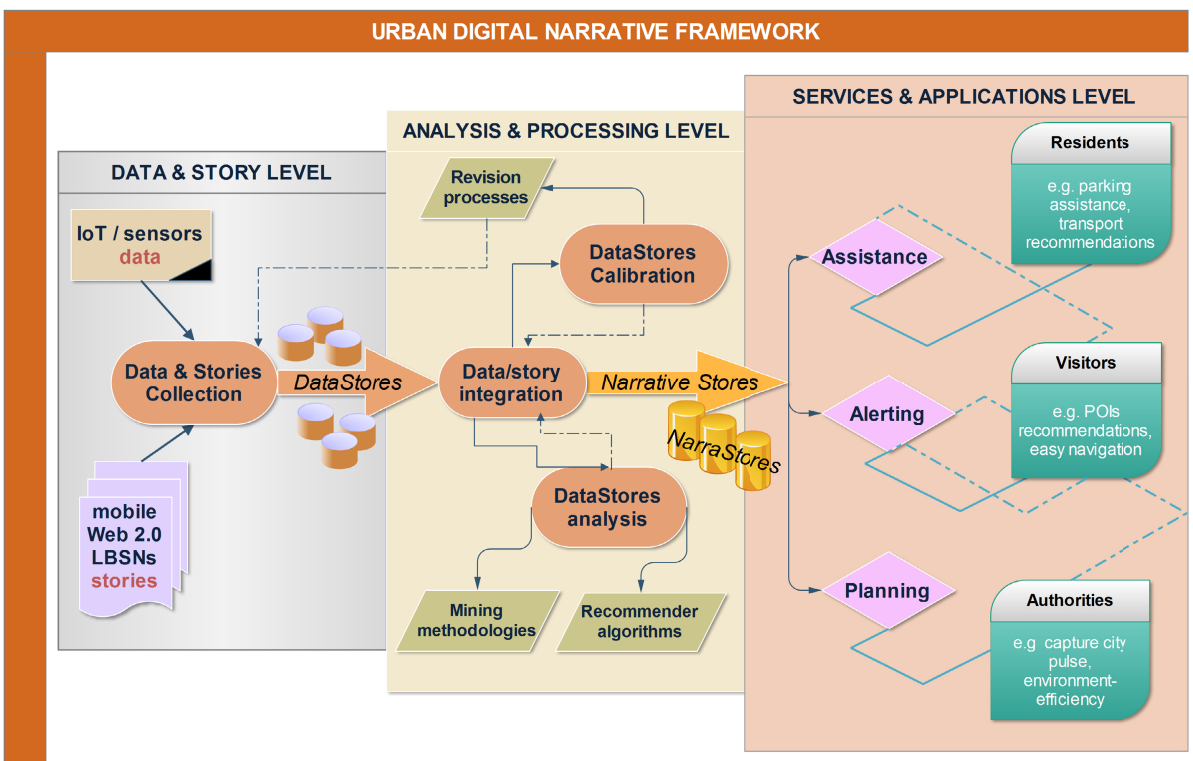

Fig. 2. A smart urban narrative aware design framework

\section{Challenging the Design: An Urban Mobility Scenario}

The narrative-aware design framework proposed herein is naturally tailored to the urban context, which is characterized by the need for flexible, scalable and adaptive processes in future Internet settings.

This section describes examples of urban applications based on the design framework, and then applies these examples to a specific event, namely a jazz festival. It is assumed that the urban context is one of a highly populated city with smart sensor installations at various locations (parking places, city lights, transport 
buses etc). The fictitious city in this case is known as "SmartVille". SmartVille would also have in place a dedicated City Department, whose objective would be to support narrative-aware services under the proposed design framework.

\subsection{Examples of Narrative-Aware Urban Applications}

SmartVille hosts and supports scalable DataStores and the resulting NarraStores, which are repositories stored in large scale data centers. As described in the previous section, these NarraStores include both SmartVille's physical data (generated by sensors and IoT installations) and its social data streams (generated by residents on the move). The NarraStores collect social data in an anonymous manner, such that no personal or private data is traceable. These NarraStores are then offered by city authorities either for public sector use or for private enterprises, for the development urban applications and services.

Table 2. An indicative list of smart narrative-aware services

\begin{tabular}{|l|l|l|l|l|}
\cline { 3 - 5 } \multicolumn{2}{l|}{} & \multicolumn{3}{c|}{ Narrative stores Processes } \\
\hline Service & Description & Integration & Analysis & Calibration \\
Park & $\begin{array}{l}\text { Monitors parking slots and } \\
\text { recommends alternatives; } \\
\text { Offered by the City }\end{array}$ & Real-time & & $\checkmark$ \\
\hline $\begin{array}{l}\text { City- } \\
\text { Watch }\end{array}$ & $\begin{array}{l}\text { Emergency alerting } \\
\text { weather, fire, etc); } \\
\text { Offered by the City }\end{array}$ & Real-time & $\checkmark \checkmark$ & $\checkmark \checkmark \checkmark$ \\
\hline $\begin{array}{l}\text { City- } \\
\text { Nav }\end{array}$ & $\begin{array}{l}\text { City Navigation opportu- } \\
\text { nities in a eco-friendly } \\
\text { manner; Offered by } \\
\text { mobile vendors }\end{array}$ & Asynchronous & $\checkmark \checkmark \checkmark$ & $\checkmark$ \\
\hline $\begin{array}{l}\text { City- } \\
\text { Pulse }\end{array}$ & $\begin{array}{l}\text { Capture residents opinions } \\
\text { and moods; Offered by } \\
\text { mobile vendor to the } \\
\text { authorities }\end{array}$ & Asynchronous & $\checkmark \checkmark \checkmark$ & $\checkmark \checkmark \checkmark$ \\
\hline
\end{tabular}

The city's NarraStores are made available to these public and private clients through a cloud infrastructure, as follows:

- Resident cloud services: Resident-specific information regarding daily urban living, public places and things with common safety concerns (in coordination with city police and other related departments). Costs are sponsored by the City and services are therefore offered to residents on a discounted basis.

- Emergency cloud services: Emergency information and services available to all residents, visitors and the wider general public over the Internet and mobile networks (free of charge) 
- Public cloud services: Information, navigation and emergency services available to residents, visitors and the wider general public over mobile networks and the Internet (free or offered on a pay-per-usage model).

- Private cloud services: offered to private organizations (e.g. mobile or Internet vendors) to encourage the development of advanced and innovative city services (at market cost).

A number of services and applications in Smartville use the cloud infrastructure. Four examples are set out in Table 2 below. Technology developers and providers harvest the City's NarraStores to create new services and applications. They use the narrativeaware design processes (e.g. analysis, calibration) to constantly improve and enhance their offerings. Table 2 sets out the type of integration (real-time or asynchronous), and the amount of analysis and calibration required for the service in question.

For example, the SmartVille City-Watch service uses the integrated NarraStores, such that emergencies are reported in realtime and in context, through both physical and social data. Such emergencies are captured by peak activities recorders at the sensor end (e.g. excessive heat) verified by simultaneous social bursts (e.g. heavy tagging on fire) through Web 2.0. Careful analysis (tag-based clustering) and algorithms (collaborative filtering), are applied to the DataStores in order to alert users of emergencies.

Another example is the SmartVille City-Pulse service, which monitors NarraStore content by continuously analyzing and calibrating DataStores content asynchronously (i.e. not necessarily in real-time). It uses appropriate time-aware clustering on the DataStores (to reveal sensor/social groups) to make cluster-based recommendations (e.g. in the form of tag topic clouds). As a result, City-Pulse reports the most prominent topics highly ranked by residents during a particular time period.

\subsection{Narrative-Aware Applications in Action: The Jazz Festival}

Events like festivals and concerts are quite popular in SmartVille. For instance, the city hosts an annual 3-day jazz festival, attracting many residents and visitors who use the services highlighted above: City-Watch, City-Park, City-Nav and City-Pulse.

Table 3. Use of narrative aware services in practice

\begin{tabular}{|l|l|l|l|l|}
\hline & City-Park & City-Watch & $\begin{array}{l}\text { City- } \\
\text { Nav }\end{array}$ & City-Pulse \\
\hline pre-event arrangements & $\checkmark \checkmark \checkmark$ & $\checkmark \checkmark \checkmark$ & $\checkmark \checkmark \checkmark$ & $\checkmark \checkmark \checkmark$ \\
\hline at-event assistance & & $\checkmark \checkmark \checkmark \checkmark \checkmark \checkmark$ & & $\checkmark \checkmark \checkmark$ \\
\hline post-event facilitating & $\checkmark \checkmark \checkmark \checkmark \checkmark \checkmark$ & & & $\checkmark \checkmark \checkmark$ \\
\hline future-event planning & $\checkmark \checkmark \checkmark$ & $\checkmark \checkmark \checkmark$ & $\checkmark \checkmark \checkmark$ & $\checkmark \checkmark \checkmark \checkmark \checkmark \checkmark$ \\
\hline
\end{tabular}

During the jazz festival, there are scheduled shows and concerts on various stages around the city, e.g. in the SmartVille stadium at two major city squares. Locations are equipped with IoT sensor installations and are not far from main traffic routes. 
In this context, the proposed narrative-aware services can support the jazz festival in all of its phases and create a more pleasant and engaging experience for the festival goer.

The need for each service at different phases of event planning is highlighted by the number of checkmarks in Table 3. The analysis is based on the following:

- City-Park : recommends parking spots on the basis of relevant data tracked by sensors; recommendations are delivered to festival attendants arriving or departing from festival sites, on the basis of location and tagging reports (captured by their mobile LBSN);

- City-Watch issues alerts when both sensors and social bursts report emergency situations within the festival environs; these alerts are of importance prior, during and after the festival, since they can monitor the entire span from sensor to social threads (e.g. from temperature levels to overcrowding, respectively);

- City-Nav delivers navigation recommendations (primarily) to visitors for an ecoaware and safe arrival/departure at the festival sites;

- City-pulse stores and monitors narratives during the entire festival through the fusion of sensors and social stories. As a result of this continuous processing, the narratives offer valuable information to authorities. There is a triage and ranking of narratives such that important conclusions can be reached after, during and after the event. For example, authorities might reorganize parking areas and re-program sensor installations in line with user demand for parking. It might also be possible to verify whether certain sensors are malfunctioning (e.g. sensor oversensitivity), as sensor data can be refined or even contradicted by social storytelling.

The design framework remains abstract in its main design principles in order to offer a wide range of potential uses and scenarios. Its simplicity can supports different applications and services which might range from the event managing and scheduling to new policy making.

\section{Conclusion}

Urban environments offer a fertile ground for developing and testing new smart applications in line with the Internet of Things and the future Internet vision. The narrative-aware design framework proposed herein exploits sensor and social data collection in a holistic manner through its design integration, analysis and calibration processes. The design includes qualitative data stores (and not merely quantitative ones) which embed both machine (sensors) and human (social) measurements. Alerting, assistance and planning are considered vital services in a city context, as highlighted in the event-based scenario. Narrative-aware design can be of tremendous benefit to primary future Internet city actors (residents, visitors and authorities) for a wide range of services and requirements (e.g. time-critical, long-term analysis, processing rates etc). Such a holistic approach is invaluable for the development of the smart, context-aware and user-centric services that lie at the very heart of a future Internet.

Open Access. This article is distributed under the terms of the Creative Commons Attribution Noncommercial License which permits any noncommercial use, distribution, and reproduction in any medium, provided the original author(s) and source are credited. 


\section{References}

1. Atzoria, L., Ierab, A., Morabitoc, G.: The Internet of Things: A survey. Computer Networks 54(15), 2787-2805 (2010)

2. Breunig, M., Baer, W.: Database Support for Mobile Route Planning Systems. Computers, Environment and Urban Systems 28(6), 595-610 (2004)

3. Castillo, L., et al.: SAMAP: An User-Oriented Adaptive System for Planning Tourist Visits. Expert Systems with Applications 34(2), 1318-1332 (2008)

4. Chiu, D.K.W., Leung, H.F.: Towards Ubiquitous Tourist Service Coordination and Integration: a Multi-Agent and Semantic Web Approach. In: International Conference on Electronic Commerce, pp. 574-581 (2005)

5. Driver, C., Clarke, S.: An Application Framework for Mobile, Context-Aware Trails. Pervasive and Mobile Computing 4(5), 719-736 (2008)

6. Giannopoulos, G.A.: The application of information and communication technologies in transport. European Journal of Operational Research 152 (2004); [Guinard10] Guinard, D., Trifa, V., Wilde, E.: A resource oriented architecture for the Web of Things. In: Internet of Things (IOT) Conference (December 2010)

7. Guinard, D., Trifa, V., et al.: Interacting with the SOA-Based Internet of Things: Discovery, Query, Selection, and On-Demand Provisioning of Web Services. IEEE Transactions on Services Computing 3(3), 223-235 (2010)

8. Kenteris, M., Gavalas, D., Economou, D.: An Innovative Electronic Tourist Guide Application. Pervasive and Ubiquitous Computing 13(2), 103-118 (2009)

9. Hernández-Muñoz, J.M., Bernat Vercher, J., Muñoz, L., Galache, J.A., Presser, M., Hernández Gómez, L.A., Pettersson, J.: Smart Cities at the Forefront of the Future Internet. In: Domingue, J., et al. (eds.) FIA 2011. LNCS, vol. 6656, pp. 447-462. Springer, Heidelberg (2011)

10. Niaraki, A.B., Kim, K.: Ontology Based Personalized Route Planning System Using a Multi-Criteria Decision Making Approach. Expert Systems with Applications 36(2p1), 2250-2259 (2009)

11. Prete, L.D., Capra, L.: diffeRS: A Mobile Recommender Service. In: Proceedings of the 2010 Eleventh International Conference on Mobile Data Management (MDM 2010), pp. 21-26. IEEE Computer Society, Washington (2010)

12. Sadoun, B., Al-Bayari, O.: Location Based Services Using Geographical Information Systems. Computer Communications 30(16), 3154-3160 (2007)

13. Sandholm, T., Ung, H.: Real-time Location-aware Collaborative Filtering of Web Content. In: Proceedings of the 2011 Workshop on Context-awareness in Retrieval and Recommendation (CaRR 2011), pp. 14-18. ACM, New York (2011)

14. Scherp, A., Boll, S.: Generic Support for Personalized Mobile Multimedia Tourist Applications. In: ACM International Conference on Multimedia, pp. 178-179 (2004)

15. Takeuchi, Y., Sugimoto, M.: CityVoyager: An Outdoor Recommendation System Based on User Location History. In: Ma, J., Jin, H., Yang, L.T., Tsai, J.J.-P. (eds.) UIC 2006. LNCS, vol. 4159, pp. 625-636. Springer, Heidelberg (2006)

16. Ye, M., Yin, P., Lee, W.-C.: Location recommendation for location-based social networks. In: Proceedings of the 18th SIGSPATIAL International Conference on Advances in Geographic Information Systems (GIS 2010), pp. 458-461. ACM, New York (2010)

17. Yu, C.C., Chang, H.P.: Personalized Location-Based Recommendation Services for Tour Planning in Mobile Tourism Applications. In: Di Noia, T., Buccafurri, F. (eds.) EC-Web 2009. LNCS, vol. 5692, pp. 38-49. Springer, Heidelberg (2009)

18. Zheng, Y., Zhang, L., Xie, X.: Recommending friends and locations based on individual location history. ACM Trans. Web 5(1) (2011) 